In January 1979 the 10 subjects were given a list which contained the names of the eight drugs mentioned at the promotional lunches paired with a compound having a similar trade name or a similar pharmacological action. The subjects were asked to identify the drug which had been promoted.

The average attendance of the 10 subjects was 6.4 meetings. They were able to recall correctly only $3 \cdot 1$ of the drugs they had seen promoted. The number of drugs omitted was therefore 3.3. However, they were incorrect on $2 \cdot 2$ occasions (drugs mistakenly thought to have been discussed). Thus the subjects studied had forgotten more drugs than they had remembered (even when confronted by the names), and a third of the drugs marked had not been promoted. If these results are a measure of advertising success then we suggest that this type of promotion has severe limitations.

The sale of a drug is the criterion of success. The chief pharmacist did not note an increase in prescriptions following any of the eight meetings. Each lunch cost the promoting company $£ 110$. It may be that intangibles such as the maintenance of friendly relations between the companies and the profession is value for money. But $£ 880$ appears to have purchased little in our hospital. Such a sum would be about three months' salary for a junior research technician or would buy or aid purchase of laboratory apparatus. Would this be better value for money?

JANET BUCK ROGER GABRIEL

St Charles's Hospital

St Char $106 \mathrm{DZ}$

\section{Coronary artery spasm}

SIR,-Your interesting leading article (14 April, p 969) prompts me to remind readers of the value of the Müller manoeuvre to relieve this potentially dangerous condition.

In this method the patient is persuaded to attempt to inhale with the nose and mouth closed, thereby creating a partial vacuum in the mouth and in the lungs around the heart. Under such conditions blood is drawn into the coronary arteries against the spasm and chemical conditions creating the spasm may be swept away. A simpler instruction is to bid the patient to suck his finger hard, for if the glottis remains open then "when you suck your finger you suck your heart just as hard."

If confirmation for such a procedure is needed let mouth suction be applied to an area of the skin of the hand which has been blanched by a small injection of adrenalin. At $60 \mathrm{~mm} \mathrm{Hg}$ suction the area is seen to flush as the arterioles are opened and normal circulation to be restored in a few minutes in an area which would otherwise remain vasoconstricted for some 20 minutes. Measurements on a patient performing the Müller manoeuvre during cardiac catheterisation show an increase in coronary flow transiently, but of course no increase in cardiac output, since the heart must work (contract) against the suction pressure. ${ }^{1}$

As a general practitioner called only too frequently to patients with severe angina and sometimes collapsed with apparent infarct, I can state that in about $50^{\circ}$ o of cases symptoms are immediately relieved by this technique. The suction need only be maintained for two or three seconds, and may be repeated as circumstances dictate.

If these observations are substantiated by others it then follows that the exercise could be performed for a few seconds a day as a prophylactic regimen. The suction should not be maintained for longer than a few seconds at a time, since distension of the atria is to be avoided.

I am given to understand, although I cannot confirm it, that the method has been used in Germany, and in the past in Scotland, and favourable claims have been made for it. It would be profitable to hear from physicians who have tried using the Müller technique for the relief of coronary spasm or angina.

Ashford, Kent TN23 1QG

C N SMYTH

'Smyth, C N, Lancet, 1969, 2, 657.

\section{Unprocessed bran causing intestinal} obstruction

SIR,-The possibility that there is a critical amount of bran that should be ingested each day in the treatment of the fibre deficiency diseases has been raised by the report of Drs J Y Kang and Dr W F Doe (12 May, p 1249) showing that excessive unprocessed bran may cause intestinal obstruction. Patients often complain of abdominal cramps when first treated with bran, and the type of bran dosage and regularity of ingestion have to be altered.

My recent surgical experience in Bihar State, North India, suggested that large-bowel obstruction, predominantly due to sigmoid volvulus, was caused by an excessively highfibre diet. The Biharis ate one large meal a day composed primarily of unpolished rice and vegetables. Patients presenting with volvulus rarely complained of constipation, but often gave a history suggestive of acute or chronic volvulus. The twisted colon at operation was always free of faeces.

All patients requiring unprocessed bran should be warned against excessive administration.

JOHN RENNIE

Professorial Department of Surgery,

Charing Cross Hospital,

London W6 8RF

\section{New approach to treatment of recent stroke?}

SIR,-With respect we would refer to the letter by Dr G D Perkin (12 May, p 1283) in which he criticises this company's recent promotional literature on the use of our product Praxilene for acute stroke illness.

Dr Perkin's examination of our literature must obviously have been of a cursory and perfunctory nature before writing to you. While the brochure in question does include descriptions of two patients admitted to the stroke unit in Sheffield, we were careful to include the dates of admission and discharge so that there could not possibly be any confusion in the minds of readers-making it clear that the two patients described did not form any part of the double-blind clinical trial reported by Dr A K Admani (16 December, p 1678) but merely provided a visually interesting feature of the brochure, in the same way that we illustrate empty beds on the last page. The precise dates given for the two patients illustrated are clearly shown in the brochure as: admitted 21 September 1978, discharged November 1978; admitted 18 September 1978, discharged 14 November 1978. It can be quite readily seen that these dates were quite some time after the stated period of the trial, 16 months from June 1976. A simple calculation by Dr Perkin would have readily demonstrated this fact.

Dr Perkin compounds his confusion in the last paragraph of his letter by referring to a letter published in the $B M \mathcal{F}$ (10 February, $p$ 412) from his hospital criticising $\mathrm{Dr}$ Admani's work, maintaining that we did not understand or had not considered the merits of that criticism. Examination of the brochure clearly shows the date of printing to be January 1979.

In view of the frivolous criticism of this company made by Dr Perkin we would not be out of order in expecting an apology.

Lipha Pharmaceuticals Limited,

D R GIFFORD West Drayton, Middx UB7 7LD

\section{Genitofemoral neuropathy}

SIR,-Dr M D O'Brien described (21 April, $p$ 1052) an interesting case of the effect of pressure on the femoral branch of the genitofemoral nerve. He stated, as is often taught in anatomy textbooks, that the genital branch of this nerve enters the inguinal canal through the deep inguinal ring.

However, the genital branch, which supplies the cremaster muscle and the skin of the groin and scrotum, accompanied by the cremaster artery and veins, is observed at operations for inguinal hernia to enter the inguinal canal not through the deep inguinal ring but through the posterior inguinal wall about $1 \mathrm{~cm}$ medial to the ring at the upper border of the iliopubic tract (the deep femoral arch). ${ }^{1}$ The genital branch and cremaster vessels are thus free from injury when the ring is repaired for oblique inguinal hernia. In repair of direct inguinal hernia which takes place medial to the ring the nerve and vessels are in danger of injury by needle puncture or compression by tight sutures. It is often of value to excise a full length of the nerve and vessels to obtain a clear field for this operation.

W J LytLe

Sheffield S10 3LS

${ }^{1}$ Lytle, W J, Annals of the Royal College of Surgeons of England, 1951, 9, 245.

\section{Overseas aid-urban hospitals or} primary care?

SIR,-Dr A W Williams (12 May, p 1286) stresses the need for Third World countries to integrate preventive and curative medicine in their health services. No one could possibly disagree. However, the problem that most such countries face is that between $60 \%$ and $80 \%$ of their health budget is already spent on hospital services, often to the detriment of prevention and primary care.

Large referral hospitals in cities are often equipped with most of the technology of western medicine, while dispensaries and health centres run out of chloroquine and isoniazid. In theory, the large hospitals act as referral centres for the surrounding population, but the lack of facilities for transferring patients makes such a system difficult to operate in practice. Of the patients admitted to the main referral hospital in Dar-es-Salaam, Tanzania, $96 \%$ come from within the city boundaries. Referral hospitals often act as training centres for medical auxiliaries, but is it not more appropriate to train health workers 